\title{
Representación contable desde la perspectiva del impacto ambiental empresarial. En el contexto del desarrollo de actividades industriales en Latinoamérica*
}

doi:10.11144/Javeriana.cc16-41.rcpi

\section{Loyda Colmenares}

Doctora en ciencias gerenciales. Profesora asociada, coordinadora de la carrera de Contaduría Pública, Universidad de Los Andes (ULA), Núcleo Rafael Rangel, Trujillo, Venezuela. Investigadora del Centro Regional de Investigación Humanística, Económica y Social (CRIHES). Programa de Estímulo a la Innovación e Investigación, PEII Nivel B. Correo electrónico: loydacdc@ula.ve

\section{Rolando Adriani}

Doctor en ciencias gerenciales. Profesor asociado, jefe del Departamento de Ciencias Económicas, Administrativas y Contables, Universidad de Los Andes (ULA), Núcleo Rafael Rangel, Trujillo, Venezuela. Investigador del Centro Regional de Investigación Humanística, Económica y Social (CRIHES). Programa de Estímulo a la Innovación e Investigación, PEII Nivel A.

Correo electrónico: adriani@ula.ve

\author{
Yosman Valderrama \\ MSc en gerencia empresarial. Licenciado en contaduría \\ pública. Profesor ordinario, Coordinador del Área Con- \\ table, Universidad de Los Andes, Núcleo Rafael Rangel, \\ Trujillo, Venezuela. Gerente de Servicios Contables y \\ Financieros en Valderrama Barrios \& Asociados. \\ Correo electrónico: yosmanjose@ula.ve
}

\footnotetext{
* Artículo de investigación científica derivado de la tesis doctoral Normativas contables y gestión ambiental en empresas extractivas, presentado por Loyda Colmenares, ante la Universidad Privada Doctor Rafael Belloso-Chacín, URBE, el 23 de julio de 2011, y a partir de la cual los investigadores profundizaron en los constructos teóricos examinados para concluir sobre el contexto latinoamericano.
} 
Resumen Las tendencias actuales de la representación contable empresarial apuntan al registro de hechos o fenómenos contables derivados de diversas perspectivas, entre las que se encuentran la ecológica o ambiental; en este sentido, además de registrar los resultados económicos y financieros de las entidades, la ciencia contable se enfrenta al reto de enfocarse en la valoración y presentación de datos derivados del impacto empresarial en el ecosistema. Con esta concepción, la investigación abarcó como objetivo analizar la representación contable desde la perspectiva del impacto ambiental originado por el desarrollo de actividades industriales en Latinoamérica. Metodológicamente, el estudio se llevó a cabo por medio de una exploración documental, utilizando como técnicas el fichaje, el análisis crítico y el resumen analítico. Entre las reflexiones finales, la revelación de información económica y financiera derivada de la actuación industrial en el ecosistema se sustenta desde la óptica ecológica en la cuantificación del impacto ambiental para presentarse como parte integral del lenguaje financiero de las entidades, amparados en el supuesto de negocio en marcha y revelación suficiente de la contabilidad, los cuales indican que los estados financieros deben revelar cualquier hecho que pueda poner en duda la situación financiera de la empresa o la prosecución de su giro económico.

Palabras clave representación contable; impacto ambiental; empresas industriales

Código JEL M41, Q56, L29

\section{Accounting Representation from the Corporate Environmental Impact Perspective. In the Context of the Development of Industrial Activities in Latin America}

\footnotetext{
Abstract Current tendencies for corporate accounting
} representation aim to record accounting facts or phenome- na derived from different perspectives, among which we find the ecological or environmental one. In that sense, in addition to record the financial and economic results from companies, accounting science faces the challenge related to focusing on appraising and showing data from the corporate impact on the ecosystem. With this concept, the objective of this research was to analyze the accounting representation from the perspective of the environmental impact caused by the development of industrial activities in Latin America. Methodologically, the study was carried out by means of a document exploration using techniques such as discovery, critical analysis, and analytic summary. Among the final reflections, the disclosure of economic and financial information derived from industrial actions on the environment is supported, from an ecological point of view, on the quantification of the environmental impact to be presented as an integral part of the corporate financial language. This idea is supported by the assumptions of going concern and sufficient disclosure, which state that financial statements must disclose any fact that could cause doubt about the financial situation of the company or the continuation of its line of business.

Keywords accounting representation; environmental impact; industrial companies

\section{Representação contábil a partir da perspectiva do impacto ambiental empresarial. No contexto do desenvolvimento de atividades industriais na América Latina}

Resumo As tendências atuais da representação contábil empresarial apontam para o registro de fatos ou fenômenos contábeis derivados de diversas perspectivas, entre as que se encontram a ecológica ou ambiental. Neste sentido, além de registrar os resultados econômicos e financeiros das entidades, a ciência contábil se vê desafiada a focalizar o valor e apresentação de dados derivados do impacto empresarial no ecossistema. Com esta concepção, a pesquisa 
teve como objetivo analisar a representação contábil a partir da perspectiva do impacto ambiental originado pelo desenvolvimento de atividades industriais na América Latina. Metodologicamente, o estudo se realizou através de uma exploração documental, utilizando como técnica a elaboração de fichas, a análise crítica e o resumo analítico. Entre reflexione-as finais, a revelação de informação econômica e financeira derivada da atuação industrial no ecossistema sustenta-se desde a óptica ecológica na quantificação do impacto ambiental para apresentar-se como parte integral da linguagem financeira das entidades, amparados no suposto de negócio em marcha e revelação suficiente da contabilidade, os quais indicam que os estados financeiros devem revelar qualquer fato que possa pôr em dúvida a situação financeira da empresa ou a prosecución de seu giro econômico.

Palavras-chave representação contábil; impacto ambiental; empresas industriais

\section{Introducción}

A partir de la presentación y publicación, por primera vez en Italia del tratado de contabilidad conocido como Partida Doble (efectuado por Fray Luca Pacioli, 1494), la evolución de la Contabilidad ha sido constante, al tratar de ajustarse a las demandas de la realidad organizacional, e indudablemente se han hecho aportes al desarrollo de la vida económica de la sociedad hasta el presente.

Como lo señala Leandro Cañibano-Calvo (2000), el concepto de contabilidad ha sufrido una evolución en consonancia con las líneas de investigación predominantes en los distintos momentos del tiempo. Añade el autor, que la contabilidad "es una ciencia de naturaleza económica que tiene por objeto producir informa- ción para hacer posible el conocimiento pasado, presente y futuro de la realidad económica en términos cuantitativos en todos los niveles organizativos, mediante la utilización de un método específico apoyado en bases suficientemente contrastadas, con el fin de facilitar la adopción de las decisiones financieras, de la planificación y de control interno".

En este sentido, la contabilidad hace posible que la información financiera de las organizaciones responda a pautas comunes y que estas midan sus resultados de manera un tanto más ejemplar, al tratar de proporcionar confiabilidad. Actualmente, en pleno siglo XXI, se espera alcanzar una armonización contable internacional efectiva, e introducir en la información contable-financiera los nuevos recursos económicos generadores de beneficios, de mayor valor para la organización.

Para ello, la contabilidad debe ser enfocada bajo el esquema del conocimiento científico aplicado al registro y control de las operaciones efectuadas por una entidad, que modifican su patrimonio de manera cuantitativa y cualitativa, a fin de interpretar sus efectos, lo que permite conocer los resultados de las operaciones y la situación financiera de una organización. En este contexto, es necesario involucrar la medición del impacto de la actividad industrial sobre el ambiente que, por tanto, debe tener un tratamiento contable específico, para que se puedan establecer con claridad y objetividad ciertos parámetros de registros contables para el ámbito ambiental que puedan ser valuados e informados, a partir de las tendencias contables actuales; desde el marco conceptual y la normativa establecida. 
De esta manera, Rob Gray, Jan Bebbington y Diana Walters (2006) señalan que es importante adaptar los procedimientos de contabilidad a los objetivos del desarrollo sostenible, para que se establezca un programa de valoración del ecosistema y disposiciones ambientales que cubran las expectativas del ámbito de procedimiento contable. Añaden estos autores, que si la normativa contable-financiero-ambiental no incluye cuestiones vinculadas con activos, pasivos, costos ambientales, capitalizaciones de estos e información ambiental, se podría estar subvalorando los impactos generados por las empresas al ambiente.

Asimismo, como lo señalan Nayibe Ablan y Elías Méndez (2004, p. 14), "la contabilidad, al igual que otras disciplinas científicas, no puede permanecer al margen de la problemática ambiental, pues debe contribuir a su estudio y solución, lo cual significa, en esencia, una participación activa en el contexto del desarrollo sostenible".

Bajo esta concepción, a la contabilidad le correspondería implementar un sistema de medición y valoración de los elementos naturales. Surge así la necesidad de valorar económicamente elementos específicos del ecosistema, por lo cual la cuantificación del valor económico del medio ambiente y del costo de su deterioro brindaría un instrumento útil para abordar problemas referidos a estos temas.

Por ello, ante el surgimiento de nuevas disciplinas en todas las áreas del conocimiento cuyos postulados sostienen principios que impulsan a tomar parte activa en la protección y conservación del medio ambiente, las organizaciones no pueden permanecer al margen, sino al contra- rio considerar la incorporación del uso o agotamiento de bienes y servicios ambientales en su contabilidad, cuya premisa ha originado tendencias contables vinculadas al tema; entre ellas se pueden señalar la contabilidad ambiental, como la denominan Nayibe Ablan y Elías Méndez (2004), Rob Gray, Jan Bebbington y Diana Walters (2006); por otro lado, Gabriel Rueda-Delgado (2002) y Graciela Scavone (2002) la designan contabilidad medioambiental.

Desde el enfoque económico, la contabilidad ambiental se ha especializado y han surgido inevitablemente unos subsistemas que Rob Gray, Jan Bebbington y Diana Walters (1999), citados por Mauricio Gómez-Villegas (2009, p. 61), describen como: "contabilidad de costos ambientales y contabilidad financiera ambiental, al mismo tiempo, el surgimiento de la auditoría medioambiental, sistemas de gestión medio ambiental, auditoría de gestión y de cumplimiento medioambiental, pólizas de seguros por riesgos ambientales y la auditoría financiera medioambiental".

En este contexto, la propuesta principal de la Organización de las Naciones Unidas (2002) es hacer que la contabilidad establezca mecanismos de registros contables ambientales, razón por la cual, esta organización mundial ha diseñado e implementado el Sistema de Contabilidad Ambiental y Económica Integrada, cuyo propósito principal es incluir las variables ambientales en los sistemas de contabilidad en todos sus países miembros. En efecto, se propone la preservación y conservación de los bienes tanto públicos como privados, que tengan efecto e impacto en sus políticas económicas mundiales. 
REPRESENTACIÓN CONTABLE / L. COLMENARES, R. ADRIANI, Y. VALDERRAMA / 263

Con base en estos planteamientos, la presente investigación centró su objetivo en analizar la representación contable desde la perspectiva del impacto ambiental originado por el desarrollo de actividades industriales, cuyo desempeño económico ha requerido el uso del capital natural. El estudio se estructuró en cuatro secciones: la primera presenta los aspectos metodológicos que condujeron el estudio de la variable; la segunda enuncia algunas fundamentaciones que originan la representación contable del impacto ambiental; la tercera desarrolla algunas puntualidades en el registro contable de partidas vinculadas con el ecosistema; y la cuarta enfatiza en algunas reflexiones sobre la problemática contable abordada.

\section{Aspectos metodológicos}

El estudio se llevó a cabo mediante la utilización de un enfoque cualitativo, en función de analizar la representación contable desde la perspectiva del impacto ambiental originado por el desarrollo de actividades industriales, tomando como caso de análisis el contexto latinoamericano. En este sentido, la investigación abordó los supuestos teóricos establecidos sobre la representación contable del impacto ambiental; para ello, recurre a la revisión de la fundamentación teórica de la variable objeto de estudio. El método de análisis aplicado se estructuró en tres pasos: 1) detección de la literatura; 2) revisión de la literatura; y 3) extracción y recopilación de información de interés.

\subsection{Detección de la literatura y otros documentos}

En lo que respecta a la exploración literaria del tema de la representación contable del impacto ambiental, se acudió al uso de la revisión terciaria, la cual de acuerdo a Roberto HernándezSampieri, Carlos Fernández-Collado y Pilar Baptista-Lucio (1991, p. 25), "se trata de documentos que compendian nombres y títulos de revistas y otras publicaciones periódicas". Para esto se acudió al uso de buscadores en línea y bases de datos de revistas científicas electrónicas, con el propósito de iniciar la exploración sobre los constructos teóricos que hablan sobre el tema de investigación e identificar los principales autores sobre el objeto de conocimiento.

Entre las fuentes de datos utilizadas, destacan las bases documentales virtuales Dialnet, SciELO, Redalyc y Saber-ULA, que representan hemerotecas científicas en línea de libre acceso que agrupan un conjunto de revistas arbitradas de América Latina, España y Portugal, de literatura científica hispana; su campo de acción es multidisciplinar, lo que facilitó la ubicación del material bibliográfico en la red. Asimismo, se acudió al uso de Google Académico, buscador electrónico utilizado con el fin de identificar los autores más citados sobre la representación contable del impacto ambiental; de este modo, se logró la ubicación de ponencias, revistas científicas, libros en línea y tesis doctorales.

\subsection{Revisión de la literatura y otros documentos}

Una vez aplicada la revisión terciaria, e identificados y localizados los aportes teóricos de expertos al tema de investigación, se profundizó 
en la consulta de la información mediante la revisión primaria de las fuentes, para lo cual se acudió al estudio y exploración de las tablas de contenido e índices analíticos de los textos seleccionados. Esto permitió reducir el número de artículos a estudiar para analizar solo aquellas fuentes documentales que satisfacían los requerimientos de información para el logro del objetivo planteado.

En este sentido, las fuentes sobre las que se realizó la recopilación literaria compren- den libros, revistas científicas, tesis doctorales y estándares internacionales. Sin embargo, la investigación planteada se proyectó abordar la problemática de la representación contable del impacto ambiental en el contexto latinoamericano se acudió al análisis de las principales fuentes estudiadas de acuerdo a su país de origen, con lo cual se consideraron autores cuyas investigaciones analizaron diversos países de Latinoamérica y las promulgaciones de organismos emisores de normas y entes regulares.

\begin{tabular}{lcr}
\hline \multicolumn{1}{c}{ Origen de la publicación } & $\begin{array}{c}\text { Número de artículos } \\
\text { consultados }\end{array}$ & (\%) \\
\hline Venezuela & 02 & 7,41 \\
Colombia & 08 & 29,63 \\
México & 05 & 18,52 \\
España & 05 & 18,52 \\
Argentina & 02 & 7,41 \\
Naciones Unidas & 04 & 14,81 \\
Consejo de Normas Internacionales de Contabilidad (IASB) & 01 & 3,70 \\
\hline Total consultado & 27 & 100,00 \\
\hline
\end{tabular}

Cuadro 1. Origen de las publicaciones consultadas

Fuente: elaboración propia (2015)

El cuadro 1 presenta de manera sistemática la bibliografía consultada de acuerdo al origen de los datos estudiados por cada uno de ellos, lo que permitió fundamentar el análisis del contexto latinoamericano, al considerar que los hallazgos de las investigaciones sobre las cuales se realizó la extracción y recopilación de la información de interés para este estudio, se concentran en países de Latinoamérica; sin embargo, se incluyeron algunos autores españoles que han realizado importantes aportes a los constructos estudiados.
Con estos lineamientos, el apoyo bibliográfico estuvo enmarcado en la revisión primaria de literatura latinoamericana disponible en la web, para analizar los puntos de investigación de diversos autores con variadas perspectivas a fin de contrastar sus ideas y preceptos teóricos. No obstante, los autores internacionales tratados en este estudio fueron seleccionados en función de las características de sus investigaciones y los contextos sobre los cuales estos generaron el conocimiento científico, se evaluaron las tendencias de sus hallazgos, los entornos sobre los que presentaron sus conclu- 
siones, y las similitudes de los autores base que dieron origen a sus fundamentos conceptuales.

\subsection{Extracción y recopilación de la} información de interés en la literatura Finalmente, la extracción y la recopilación de la información de interés para este estudio, se llevaron a cabo mediante el uso del análisis crítico, el resumen analítico y las técnicas de exploración de información teórica como lecturas previas, fichaje bibliográfico, esquemas de contenido y resúmenes. Este método fue aplicado una vez evaluada la información e identificados los autores cuyos resultados fueron relevantes y sus aportes al acervo del conocimiento científico han sido significativos para el avance del estudio de la problemática de la representación contable del impacto ambiental.

\section{Fundamentaciones que originan la representación contable del impacto ambiental}

Según Antoni Llull-Gilet (2001), la incorporación de la problemática ambiental a la investigación contable data aproximadamente de mediados de los años 80 , por lo cual se puede considerar que está en un grado de desarrollo incipiente. Desde esta perspectiva, la contabilidad, al igual que otras ciencias, no puede permanecer al margen de los aspectos inherentes al medio ambiente, pues debe contribuir a su estudio y resolución de dificultades, lo cual significa una participación activa en el contexto del desarrollo sostenible.

En cuanto al término desarrollo sostenible, se considera la definición del informe Nuestro
Futuro Común, de la Comisión Mundial sobre el Medio Ambiente y el Desarrollo (1987), que se refiere al desarrollo que satisface las necesidades del presente sin comprometer la habilidad de generaciones futuras para satisfacer sus propias necesidades. De igual manera, el desarrollo sostenible se puede entender como "un proceso de cambio social, en donde la explotación de los recursos, la orientación al desarrollo tecnológico y las reformas institucionales se realizan en forma armónica, ampliándose el potencial actual y futuro para la satisfacción de las necesidades y aspiraciones humanas" (Rueda, 2002, p. 52, citando a Henri Acselrad).

Bajo estas consideraciones, la Cumbre de la Tierra, celebrada en Rio de Janeiro, Brasil, en 1992, sostuvo un acuerdo global conocido como Agenda 21, con la participación de representantes de 178 países del mundo, a partir del cual, sus impulsores adquirieron compromisos que dan surgimiento a prácticas de contabilidad ambiental. En este marco, nació la propuesta de cuentas ambientales de la Organización de las Naciones Unidas (ONU), que se materializaron en el Sistema de Contabilidad Ambiental y Económica Integrada, SCAEI (System of Environmental-Economic Accounting, SEEA).

De acuerdo a las afirmaciones de Frank Eduardo Barraza-Caro y Marta Eliana GómezSantrich (2005), la concepción de contabilidad ambiental implícita en la propuesta de la ONU se orienta hacia un instrumento que por medio de la representación de las relaciones del desarrollo con el medio ambiente permita diseñar políticas gubernamentales económicas y ambientales, que consideren cuentas monetarias que incluyen información como gastos, costos 
ambientales y cuentas físicas que informan acerca de las características de los recursos naturales, así como su uso.

Como se ha señalado, en la Cumbre de la Tierra (1992), quedó planteado el compromiso de incorporar la contabilidad ambiental en las cuentas nacionales. Frank Eduardo Barraza-Caro y Marta Eliana Gómez-Santrich (2005) añaden que lo ideal es contar con la estructura de un subsistema de contabilidad ambiental, como elemento constitutivo de un sistema integral de contabilidad nacional, independiente en su operación, valoración y registro, interrelacionado con las cuentas nacionales y con la contabilidad o balance social por medio de indicadores previamente definidos y diseñados objetivamente, según los propósitos de evaluación.

En consecuencia, las organizaciones deben garantizar el suministro de información financiera que facilite la valoración, evaluación y control ambiental, pues de ella depende la eficiencia en el manejo de los recursos naturales, por parte del sector productivo, y aún más, si se evalúa el efecto contaminante derivado de la actividad empresarial industrial, por ser este el principal contaminador al utilizar la naturaleza como fuente de materiales e insumos y destino de sus desechos. Por lo tanto, estas organizaciones deben clasificar las erogaciones vinculadas en el manejo ambiental, teniendo en cuenta los conceptos contables establecidos en los Principios de Contabilidad Generalmente Aceptados, en las Normas Internacionales de Contabilidad (NIC) y por la necesidad de información de la contabilidad ambiental.

En efecto, como lo refiere Antoni LlullGilet (2001), los registros contables de la orga- nización, al incorporar los hechos económicos con fundamento ambiental, facilitarán indicadores de gestión ambiental en la empresa; pero para asegurar procesos con calidad ecológica, se deben articular sistemas de control tanto en la ejecución de los planes de manejo como en los registros contables, soportados mediante una auditoría periódica, con la cual se evaluarán el desempeño financiero y los resultados o impactos ambientales en la organización, responsables de los costos socioambientales.

\section{Puntualidades de la representación contable del impacto ambiental}

Desde la perspectiva ambiental, se pueden generar partidas contables para registrar los hechos económicos relacionados con el capital natural, los cuales de acuerdo a autores como Antonio Rueda-Manzanares, Juan Alberto Aragón-Correa e Inmaculada Martín-Tapia (2006), Rob Gray, Jan Bebbington y Diana Walters (2006), Graciela Scavone (2002) y otros, pueden englobarse en los rubros de: (a) activos ambientales, (b) pasivos ambientales, (c) provisiones y (d) costos ambientales.

\subsection{Activos ambientales}

Según Antonio Rueda-Manzanares, Juan Alberto Aragón-Correa e Inmaculada Martín-Tapia (2006), los activos ambientales representan los bienes que sirven para la preservación, protección y recuperación ambiental. Esto incluye inventarios, propiedad, planta y equipo y diferidos, que deben ser distinguidos entre las demás categorías de activo para permitir una optimización 
en la evaluación de las labores ambientales. Por su parte, Nayibe Ablan y Elías Méndez (2004) señalan que estos son bienes e inversiones, propiedades compradas o realizadas por la empresa, con la finalidad de preservar y proteger el ambiente y minimizar los daños que se le pudieran ocasionar.

No obstante, la óptica de Scavone (2002) indica que este grupo de cuentas puede englobar la representación contable de contenedores de recolección, sistemas de recuperación de calor, filtros para la contaminación de aire, in- versión para disminución de ruido, plantas de tratamiento de aguas residuales y añade la autora que estos dependerán del tipo de negocio y de la gestión ambiental.

Sintetizando, algunas partidas contables inmersas en el rubro de los activos de una organización, que pueden ser utilizadas por las empresas para registrar los efectos económicos del ambiente, son: a) inventarios; b) inversiones; y c) propiedad, planta y equipo, los cuales se muestran en el cuadro 2 .

\begin{tabular}{ll}
\hline \multicolumn{1}{c}{ Partida } & \multicolumn{1}{c}{ Aspectos que engloba o subpartidas } \\
\hline \multirow{2}{*}{ Inventarios } & Capital natural crítico (recursos no renovables, exóticos o únicos) \\
& Capital natural (recursos renovables) \\
& Capital hecho por el hombre \\
& Componente físico en el desarrollo de la política ambiental \\
Inversiones & Efectos operativos y financieros esperados de inversiones futuras \\
& Procesos tecnológicos favorables al entorno \\
Propiedad, planta y equipo & Maquinarias y equipos que reduzcan los efectos contaminantes \\
& Patentes y equipos que busquen reducir los efectos contaminantes
\end{tabular}

Cuadro 2. Activos ambientales

Fuente: Antonio Rueda-Manzanares, Juan Alberto Aragón-Correa e Inmaculada Martín-Tapia (2006); Rob Gray, Jan Bebbington y Diana Walters (2006), adaptado por los investigadores (2014).

Inventarios, en primer lugar se considera lo planteado por Rob Gray, Jan Bebbington y Diana Walters (2006), que refieren que el enfoque de inventario se orienta a la identificación, registro, control y comunicación - probablemente mediante cantidades no financieras- de las diferentes categorías del capital natural y su agotamiento o mejora, donde se pueden identificar tres tipos de capital disponible para mantener el capital natural crítico (recursos no renovables, exóticos o únicos), el capital natural (recursos renovables), y el capital hecho por el hombre.
Para Eduardo Mantilla-Pinilla (2006), estos tipos de inventarios son un valor patrimonial de los recursos como activos naturales de una sociedad o valor de existencia del recurso; el valor de los inventarios de cada recurso lo conforman los registros en cuentas definidas de acuerdo a los beneficios generados y según las normas de clasificación; hay que tener presente que los registros se alteran por cambio en los volúmenes o extensiones que constituyen los inventarios y por alteración de las características de los recursos. 
Inversiones, siguiendo a Eduardo MantillaPinilla (2006), en las cuentas de inversión se registrarán las erogaciones que constituyen un componente físico en el desarrollo de la política ambiental. Por lo cual, estas partidas agrupan aportaciones dinerarias realizadas a la adquisición de bienes para la ejecución de programas de mejora, conservación y mantenimiento del ecosistema de donde surgirán posiblemente algunos derechos de la organización.

Por su parte, Ángel Tejada-Ponce (1999) expresa que algunas organizaciones internacionales, entre ellas el Instituto de Contabilidad y Auditoría de Cuentas, ICAC, en España, en aras de garantizar una objetividad en el reconocimiento, valoración e información de estos activos medioambientales aprobó normas que establecen pautas y lineamientos relevantes para la consideración de los aspectos ecológicos en la contabilidad de las organizaciones, además entre otros aspectos establece que, dentro de su situación fiscal, se incluirá información sobre las deducciones por inversión en medidas para reducir el impacto ambiental. Además, debe presentarse información precisa que indique los efectos operativos y financieros que se prevén como consecuencia de las inversiones futuras en este aspecto.

Propiedad, planta y equipo, según Enrique Blanco-Richart (2006), la propiedad industrial medioambiental registrará la adquisición o creación por la propia empresa de procesos tecnológicos favorables al entorno. Adicionalmente, para Nayibe Ablan y Elías Méndez (2004), este tipo de activos se emplea en la reducción de impactos ambientales producidos por las empresas.
En cuanto a la determinación de este tipo de bienes, Graciela Scavone (2002) afirma que es necesario que todos los activos sean valorados independientemente y de manera conjunta entre el gerente ambiental y los miembros del equipo de protección al ambiente, porque desde el punto de vista contable no se distingue entre activos fijos usados para los tratamientos de emisión, las tecnologías más limpias u otras propiedades del negocio.

Para Pere Sabaté-Prats (2001), la propiedad, planta y equipo se usa cuando las empresas opten por realizar la adquisición de maquinarias y equipos para reducir los efectos contaminantes, el tratamiento contable debería ser el mismo que el seguido para cualquier otro activo de características similares. Como lo refiere Danilo Ariza-Buenaventura (2000), en una organización industrial, para reducir el impacto ambiental generado por su actividad, se deberá acceder a nuevas tecnologías e implementar procesos descontaminadores; esto requerirá invertir en patentes, equipos, entrenamiento y capacitación de su personal, y será parte de sus activos (Sinopsis de la representación contable desde la perspectiva del impacto ambiental en el cuadro 6).

\subsection{Pasivos ambientales}

Según Luis Eliel Quintero y José Daniel Anido, los pasivos asociados a la protección ambiental corresponden a las obligaciones derivadas de incertidumbre. Para Graciela Scavone (2002), son aquellos que ayudan a hacer que el agente causante del daño ambiental pague por reparar el efecto causado. En tal sentido, Gabriel RuedaDelgado (2002) expresa que estos constituyen beneficios económicos, que serán sacrificados en 
función de la obligación contraída frente a terceros, para preservación y protección ambiental. Los gastos relativos al medio natural del período actual o anterior originan adquisiciones de bienes permanentes o existencia de riesgos.

En relación con este punto, Pere SabatéPrats (2001) refiere que los pasivos ambientales están constituidos por pérdidas o deudas en las que podrán incurrir las empresas como consecuencia del impacto sobre el entorno físico, aunque no llegue a conocerse el importe o momento en que sucederán.

Al mismo tiempo, Antoni Llull-Gilet (2001) especifica que la naturaleza de estas obligacio- nes es generar una salida futura de recursos. Esta puede ser: (a) jurídica, cuando la empresa tiene una obligación de carácter legal o contractual de evitar, reducir o reparar un impacto medioambiental; (b) tácita, cuando nace de compromisos asumidos por la empresa, a los que esta no puede sustraerse porque - como consecuencia de declaraciones, intenciones, principios o pautas de actuación - ha asumido una responsabilidad medioambiental frente a terceros, que la obliga a adoptar medidas para evitar, reducir o reparar los daños generados. Entre ellos se pueden nombrar: (a) pasivo cierto; (b) pasivo contingente (cuadro 3).

\begin{tabular}{llll}
\hline Naturaleza & \multicolumn{1}{c}{$\begin{array}{c}\text { Descriptor de la } \\
\text { naturaleza }\end{array}$} & $\begin{array}{c}\text { Característica } \\
\text { común }\end{array}$ & \multicolumn{1}{c}{ Partidas específicas } \\
\hline Jurídica & $\begin{array}{l}\text { Derivados por lineamientos } \\
\text { normativos establecidos }\end{array}$ & & $\begin{array}{l}\text { a) Pasivo cierto: se cuenta con datos precisos de } \\
\text { la cuantía de la obligación contraída. }\end{array}$ \\
& $\begin{array}{l}\text { Derivados de compromisos } \\
\text { asumidos por la empresa } \\
\text { ante terceros }\end{array}$ & $\begin{array}{l}\text { Evitar, reducir o } \\
\text { reparar un impacto contingente: se caracteriza } \\
\text { medioambiental }\end{array}$ & $\begin{array}{l}\text { por la existencia de incertidumbre en su } \\
\text { determinación, está condicionado a la }\end{array}$ \\
& & & $\begin{array}{l}\text { ocurrencia o no de sucesos futuros que no están } \\
\text { enteramente bajo el control de la entidad. }\end{array}$ \\
\hline
\end{tabular}

Cuadro 3. Naturaleza de los pasivos ambientales

Fuente: Antoni Llull-Gilet (2001), adaptado por los investigadores (2014)

a) Pasivo cierto. Antoni Llull-Gilet (2001) señala que este pasivo se registra, si se tiene la seguridad de que en el futuro se debe responder a una determinada responsabilidad de naturaleza medioambiental, y su vencimiento e importe son incuestionables. Por tanto, cuando se tiene una deuda real y cierta de carácter ambiental en la que no hay incertidumbre alguna en cuanto a su importe o fecha de pago, tal obligación deberá reconocerse igual que el resto de pasivos frente a terceros, razón por la cual la empresa tiene un compromiso actual cierto ocasionado por hechos pasados, que generará un flujo de salida de recursos, siendo el importe y fecha indudables.

b) Pasivo contingente. Según el Consejo de Normas Internacionales de Contabilidad (IASB, 2012), es toda obligación posible "surgida a raíz de sucesos pasados, cuya existencia quedará confirmada solo si llegan a ocurrir, o no, uno o más sucesos futuros inciertos que no están enteramente bajo el control de la entidad". La norma internacional de conta- 
bilidad 37 (NIC 37) añade que estos pasivos pueden evolucionar de manera diferente a la inicialmente esperada. Por tanto, serán objeto de revisión consecutivamente, con el fin de determinar si se ha convertido en probable la eventualidad de salida de recursos, que incorporen beneficios económicos futuros, para proceder en tal caso a su cancelación.

Particularmente, Graciela Scavone (2002) señala que en materia ambiental los pasivos pueden surgir producto de la actividad empresarial, cuyos efectos han generado: (a) contaminación de las capas de agua subterráneas (trabajar con sustancias que contienen solventes); (b) contaminación de la superficie del agua (derrames o transporte peligroso); (c) emisiones al aire (liberación repentina por rotura del equipamiento de tratamiento de la contaminación); (d) emisión de energía (emisiones radioactivas); y (e) contaminación del suelo (agua contaminada por omisión de protección).

Para finalizar, Danilo Ariza-Buenaventura (2000) destaca que lo más importante para este rubro de partidas es la problemática e incertidumbre que rodean su cuantificación. En este sentido, el llamado pasivo ambiental contingente, si existe, debe ser cuantificado aunque sea aproximadamente. Sin embargo, si su existencia está llena de una incertidumbre significativa, esta obligación debe ser referenciada al menos en notas a los estados financieros, para detallar sus aspectos cualitativos y los datos relevantes que a juicio del contador necesiten ser mostrados a los usuarios de la información financiera (Sinopsis de la representación conta- ble desde la perspectiva del impacto ambiental en el cuadro 6).

\subsection{Provisiones}

El IASB (2012) define las provisiones como "pasivos en los que existe incertidumbre acerca de su cuantía o vencimiento". Además, indica que solo en casos extremadamente particulares, en los que no será posible la estimación de la cuantía de la deuda, deben ser utilizadas para cubrir los desembolsos para los cuales fueron originalmente reconocidas, siempre y cuando se den ciertas circunstancias. La NIC 37 contempla elementos específicamente referidos al ambiente, en particular en el caso de las provisiones: multas medioambientales o costos de reparación de esos daños causados en contra de la ley, costos por el abandono de una instalación de extracción de petróleo o de una central nuclear, siempre que esté obligada a restaurar los daños ya ocasionados por su funcionamiento.

Adicionalmente, Luis Eliel Quintero y José Daniel Anido (2004) expresan que las provisiones están representadas por el aspecto ambiental; se requieren para reconocer el pasivo de futuras removilizaciones y costos de restauración del sitio, cuando la probabilidad de su ocurrencia sea establecida como resultado de una ley ambiental o porque la empresa ha establecido una política para ello. De este modo, Graciela Scavone (2002) refiere que son un instrumento clásico para anclar un esquema de protección de riesgo de la empresa en los estados financieros y deben estar formados por pasivos contingentes y pérdidas potenciales latentes de las transacciones del negocio. 
Al respecto, Jorge Tua-Pereda (2001) señala que desde el punto de vista ambiental, la empresa deberá dotar una provisión ambiental cuando: (a) esté obligada, por ley o contrato, a prevenir, reducir o reparar daños al ambiente; (b) se encuentre frente a una obligación asumida, en la que tenga el compromiso de prevenir, reducir o reparar un daño ambiental; c) cuando su política, objetivo, práctica del sector, y las expectativas públicas imponen a la dirección la necesidad de utilizar esta cuenta; y, d) cuando la empresa ha comunicado que tal provisión prevendrá, reducirá o reparará un daño al ambiente.

De acuerdo a Rob Gray, Jan Bebbington y Diana Walters (2006), en la gama de pro- visiones que pueden ser consideradas en el área medioambiental están: a) provisión para remover y reciclar los desechos; b) provisión para restauración y disposición final; y c) provisión para limpieza del sitio contaminado. No obstante, algunos otros autores, como Luis Eliel Quintero y José Daniel Anido (2004) expresan que adicional a las ya mencionadas, hay otras provisiones que se pueden identificar en una representación contable basada en el ambiente, y estas son: provisión para medidas de protección ambiental; provisión por riesgos y gastos ambientales; provisión por cese de actividades (cuadro 4).

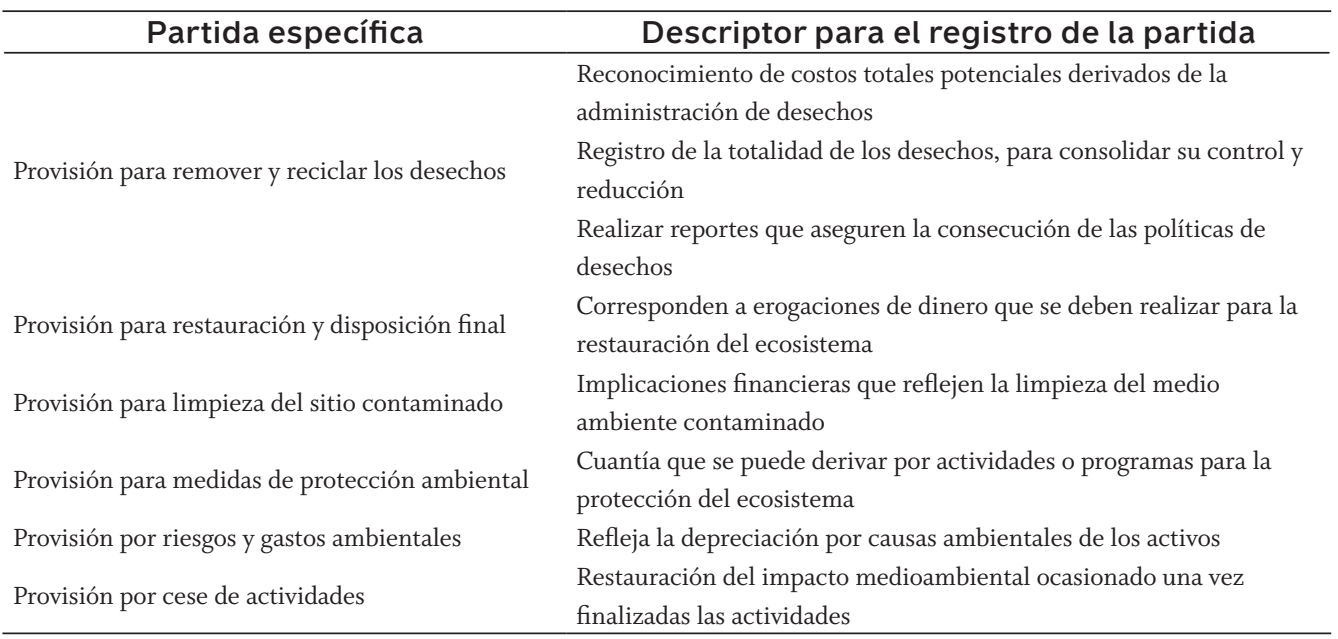

Cuadro 4. Provisiones derivadas del impacto ambiental Fuente: Rob Gray, Jan Bebbington y Diana Walters (2006), y Luis Eliel Quintero y José Daniel Anido (2004), adaptado por los investigadores (2014)

a) Provisión para remover y reciclar los desechos: según Rob Gray, Jan Bebbington y Diana Walters (2006), la administración de desechos y metas de reciclado requiere este tipo de provisión. Hay tres modalidades para contabilizarla. La primera reconoce los costos totales actuales y potenciales de la administración de desechos, por lo cual debe identificarlos, de acuerdo a la actividad. En la segunda, se establece un sistema de re- 
gistro y comunicación sobre las cantidades físicas de desechos, para registrar la totalidad de los desechos, consolidar su control y reducción. Y en la tercera, se hace una lista de desechos, se realiza una revisión inicial, se detectan responsabilidades administrativas, para minimizar y administrar los desechos, y realizar reportes que aseguren la consecución de las políticas de desechos. Por su parte, Graciela Scavone (2002) señala que, de existir una reserva en el balance con fecha límite de cumplimiento que conlleve una obligación por remoción de desechos y reciclado, los cuales surgen en intervalos periódicos, esto debe ser considerado para la creación de este tipo de provisión.

b) Provisión para restauración y disposición final: al respecto, Rob Gray, Jan Bebbington y Diana Walters (2006) consideran que es necesario identificar por separado, las erogaciones realizadas por concepto de restauración y disposición final. Y Graciela Scavone (2002) señala que estas provisiones se someten a reglas que requieren medidas integrales para restaurar el paisaje original, por ejemplo: el recorrido del río de una planta de generación eléctrica, o la demolición controlada de edificios en una planta de energía nuclear.

c) Provisión para limpieza del sitio contaminado: Rob Gray, Jan Bebbington y Diana Walters (2006) señalan la conveniencia de considerar las implicaciones financieras, en la creación de cuentas que reflejen la limpieza en los terrenos contaminados. De acuerdo a lo estimado por Graciela Scavone (2002), esta provisión debe ser creada cuando haya la posibilidad que la obligación aparezca.
Las provisiones para la limpieza de territorio contaminado están relacionadas con el suelo y con el agua.

d) Provisión para medidas de protección ambiental: Luis Eliel Quintero y José Daniel Anido (2004) exponen que para cada tipo de provisión medioambiental, y siempre que su importe sea significativo, la empresa debe informar: (a) valor en libros al principio y final del período; (b) dotaciones e incrementos en las provisiones existentes efectuadas en el período; (c) importes utilizados o no en el período; (d) aumento, durante el período, de intereses acumulados; (e) breve descripción de la naturaleza de la obligación contraída, (f) calendario de las salidas de beneficios económicos; ( $\mathrm{g}$ ) indicación de las incertidumbres relativas al importe o al calendario de las salidas de recursos que producirá la provisión.

e) Provisión por riesgos y gastos ambientales: de acuerdo a Pere Sabaté-Prats (2001), se debería crear una provisión que refleje la depreciación por causas ambientales de los activos, esta pudiera derivarse de una decisión judicial; no obstante, la mayor dificultad estriba en la estimación del importe de la hipotética sanción que pudiera imponer un juez. Agrega el autor, que en el supuesto de que no se plantee ninguna demanda judicial contra la empresa y esta modifique su estrategia al adaptar sus vertidos contaminantes a la normativa legal, no debería cancelar la provisión para riesgos y gastos, mientras sus actuaciones dolosas no hubieran prescrito e impidieran iniciar acciones de carácter legal contra sus actos pasados. 
f) Provisión por cese de actividades: al respecto, Enrique Blanco-Richart (2006) puntualiza que las empresas tienen la obligación de restaurar el impacto medioambiental ocasionado una vez finalizada sus actividades, por lo cual es recomendable que al final de cada ejercicio, se revise el saldo de la provisión y se compruebe su adecuación con el importe previsto para la restauración ( $\mathrm{Si}$ nopsis de la representación contable desde la perspectiva del impacto ambiental en el cuadro 6).

\subsection{Costos ambientales}

Considerados por Luisa Fronti de García y Carmen Fernández-Cuesta (2007), como el consumo, necesario y debidamente valorado, de factores productivos relacionados con recursos naturales indispensables para las operaciones económicas; el aprovechamiento del entorno natural de desechos, generados por las actividades de producción y consumo; y el conjunto de bienes y servicios ambientales que se orientan a las necesidades del hombre. Enrique Blanco-Richart (2006) destaca que es el sacrificio de recursos necesarios para elaborar un producto, con la posterior finalidad de incrementar su valor añadido. Si lo que se realiza afecta a los recursos naturales y la calidad de vida, se está haciendo referencia a los costos ambientales y por tanto, estos son un elemento más del costo de producción y tienen impacto en la fijación de los precios de venta.

Siguiendo a Eduardo Mantilla-Pinilla (2006), los costos ambientales se establecen como herramienta útil en la evaluación de los impactos ambientales de la actividad económica y en la medición de la sostenibilidad. Juan García-Colín (2001) indica que se relacionan con la actividad de eliminar los efectos contaminantes por gases, humo, ruido, descargas residuales, desechos sólidos o líquidos del proceso de producción; y también con la de convertir los productos terminados en artículos que sean biodegradables. El autor sostiene que es conveniente la internalización de estos costos, pues ellos afectan la vida de las empresas $y$, por consiguiente, los procesos de desarrollo económico.

Por su parte, Antoni Llull-Gilet (2001) refiere que estos costos son los derivados del consumo de los recursos naturales y de la prevención, reducción y restauración de los impactos medioambientales negativos de la actividad productiva. Por lo cual, es necesario que la contabilidad incluya en su ámbito de trabajo todos aquellos costos de carácter ambiental, esto lleva a que se contabilicen todos los costos en que la empresa incurre por el consumo de elementos, como aire, agua y recursos no renovables, entre otros. En efecto, se pueden mencionar: a) costos de apertura; b) costos de prevención; c) costos por daños; d) costos por exigencias administrativas; e) costos por fallas; y f) costos por detección (cuadro 5).

a) Costos de apertura: Enrique Blanco-Richart (2006) menciona en este punto los costos de restauración y recuperación del entorno natural, algunos de estos elementos pueden ser canteras, minas y derribo de ruinas, entre otros. 


\begin{tabular}{ll}
\hline \multicolumn{1}{c}{$\begin{array}{c}\text { Partida } \\
\text { específica }\end{array}$} & Descriptor para el registro de la partida \\
\hline $\begin{array}{l}\text { Costos de apertura } \\
\text { Costos de prevención }\end{array}$ & $\begin{array}{l}\text { Se incluyen los costos derivados por la restauración y recuperación del entorno natural } \\
\text { Derivados por la prevención de la contaminación } \\
\text { Costos por daños }\end{array}$ \\
$\begin{array}{l}\text { Costos por exigencias } \\
\text { administrativas }\end{array}$ & Constituido por los erogaciones derivadas de exigencias administrativas para la obtención de \\
Costos por fallas & permisos y demás trámites administrativos requeridos \\
Costos por detección & Corresponden a las fallas que se ocasionan durante el proceso de producción \\
& ambiental de la empresa \\
\hline
\end{tabular}

Cuadro 5. Costos derivados del impacto ambiental

Fuente: Antoni Llull-Gilet (2001), adaptado por los investigadores (2014)

b) Costos de prevención: siguiendo a Enrique Blanco-Richart (2006), estos costos se conforman por las erogaciones de dinero efectuadas para la prevención, sistemas de detección e información sobre la contaminación, y los de investigación y desarrollo. Según Ángel Tejada-Ponce (1999), son aquellos relacionados con el aseguramiento de la calidad ambiental en la organización, que incluyen los costos de análisis y evaluación (que comprenden todos los elementos que permiten determinar la posición de la organización respecto al entorno vital).

c) Costos por daños: para Ana Citlalic González-Martínez (2001), se componen de dos elementos: (a) externalidades de la recolección, separación y transporte de los desechos susceptibles de ser cuantificados; $y$, (b) externalidades resultantes del proceso mismo del reciclaje. Ángel Tejada-Ponce (1999) señala que se producen al no operar en las condiciones ambientales adecuadas. Tienen su origen en la falta o inadecuación del sistema de gestión ambiental y se clasifican en este caso como pérdidas. No obstante, estas actividades no siempre se originan por fallos en la gestión ambiental, dado que la actividad desarrollada en determinados sectores (minería, siderurgia, eléctrico, construcción y otros), pues su propio objeto es contaminante.

d) Costos por exigencias administrativas: Enrique Blanco-Richart (2006) destaca que estos pueden estar constituidos por permisos, licencias e informes periódicos remitidos a los organismos competentes, así como tributos, multas y sanciones administrativas. Al respecto, Leandro Cañibano-Calvo (2000) indica que son aquellos vinculados con beneficios medioambientales que reducen o evitan la contaminación futura, y que podrían ser registrados como gastos cuando: (a) los costos no están asociados con beneficios medioambientales futuros; (b) se imputan los costos previamente reconocidos como activos, a medida que se producen los beneficios medioambientales correspondientes; $y$, (c) se reconocen contablemente los futuros compromisos contraídos en materia ambiental, al registrar como pasivo del balance las provisiones para riesgos y gastos correspondientes.

e) Costos por fallas: según Charles T. Horngren, Srikant M. Datar y George Foster (2007), 
son aquellos que corresponden a las fallas que se ocasionan durante el proceso de producción (falla interna) o una vez que el producto ha salido de la empresa (falla externa). Los costos que se pueden atribuir a una falla interna incluyen tiempo ocioso de los equipos, materiales de mala calidad, desperdicio y rectificaciones. Los costos por fallas externas incluyen devoluciones, descuentos, garantía, costos por insatisfacción del cliente y pérdida de participación en el mercado.

Por otro lado, Don R. Hansen y Maryanne M. Mowen (2003) añaden que se incurre en costos de falla interna, cuando los productos y servicios no se ajustan a los re- querimientos de calidad, cuando esta falla se descubre antes de que el producto sea vendido o prestado el servicio. Los costos de falla externa son aquellos en que se incurre cuando los productos no se ajustan a los requerimientos después de que se vendieron o se prestó el servicio.

f) Costos por detección: siguiendo a Charles T. Horngren, Srikant M. Datar y George Foster (2007), al igual que Don R. Hansen y Maryanne M. Mowen (2003), son aquellos costos que inciden o en los cuales incurre la empresa al momento de detectar su nivel de desempeño ambiental (Sinopsis de la representación contable desde la perspectiva del impacto ambiental en el cuadro 6).

\begin{tabular}{|c|c|c|c|c|}
\hline \multirow[t]{2}{*}{ ¿Qué hacer? } & \multicolumn{4}{|c|}{$\begin{array}{l}\text { Registrar partidas contables vinculadas con la medición del impacto ambiental } \\
\text { Activos ambientales }\end{array}$} \\
\hline & $\begin{array}{l}\text { Clasificar el activo } \\
\text { ambiental: bienes } \\
\text { o propiedades } \\
\text { relacionados con el } \\
\text { ambiente }\end{array}$ & $\begin{array}{l}\text { Presentar el } \\
\text { activo ambiental } \\
\text { separado de otros } \\
\text { activos }\end{array}$ & $\begin{array}{l}\text { Indicar efectos } \\
\text { operativos y } \\
\text { financieros de } \\
\text { la inversión } \\
\text { ambiental }\end{array}$ & $\begin{array}{l}\text { Identificar } \\
\text { los activos } \\
\text { ambientales con } \\
\text { los responsables } \\
\text { de la gestión } \\
\text { ambiental }\end{array}$ \\
\hline \multirow{9}{*}{$\begin{array}{l}\text { ¿Cómo } \\
\text { hacerlo? }\end{array}$} & \multicolumn{4}{|c|}{ Pasivos ambientales } \\
\hline & $\begin{array}{l}\text { Clasificar pasivo } \\
\text { ambiental: deudas } \\
\text { con terceros } \\
\text { producto del } \\
\text { ambiente }\end{array}$ & $\begin{array}{l}\text { Contabilizar } \\
\text { pasivos } \\
\text { ambientales } \\
\text { al crearse la } \\
\text { responsabilidad }\end{array}$ & $\begin{array}{l}\text { Citar las deudas } \\
\text { en materia } \\
\text { ambiental no } \\
\text { determinadas de } \\
\text { manera fiable }\end{array}$ & $\begin{array}{l}\text { Contabilizar todo } \\
\text { compromiso } \\
\text { asumido } \\
\text { de carácter } \\
\text { ambiental }\end{array}$ \\
\hline & \multicolumn{4}{|c|}{ Provisiones } \\
\hline & & \multirow{2}{*}{\multicolumn{3}{|c|}{$\begin{array}{l}\text { cuando haya obligación por daños al ambiente } \\
\text { cuando haya obligación producto de la política } \\
\text { empresarial }\end{array}$}} \\
\hline & & & & \\
\hline & & \multicolumn{3}{|c|}{ para administración de desechos y reciclado } \\
\hline & Registrar provisión & \multicolumn{3}{|c|}{$\begin{array}{l}\text { que contenga las disposiciones para la restauración del } \\
\text { paisaje original }\end{array}$} \\
\hline & & \multicolumn{3}{|c|}{ para riesgos ambientales } \\
\hline & & \multicolumn{3}{|c|}{$\begin{array}{l}\text { para gastos ambientales por decisión judicial } \\
\text { para restaurar el impacto ambiental }\end{array}$} \\
\hline
\end{tabular}




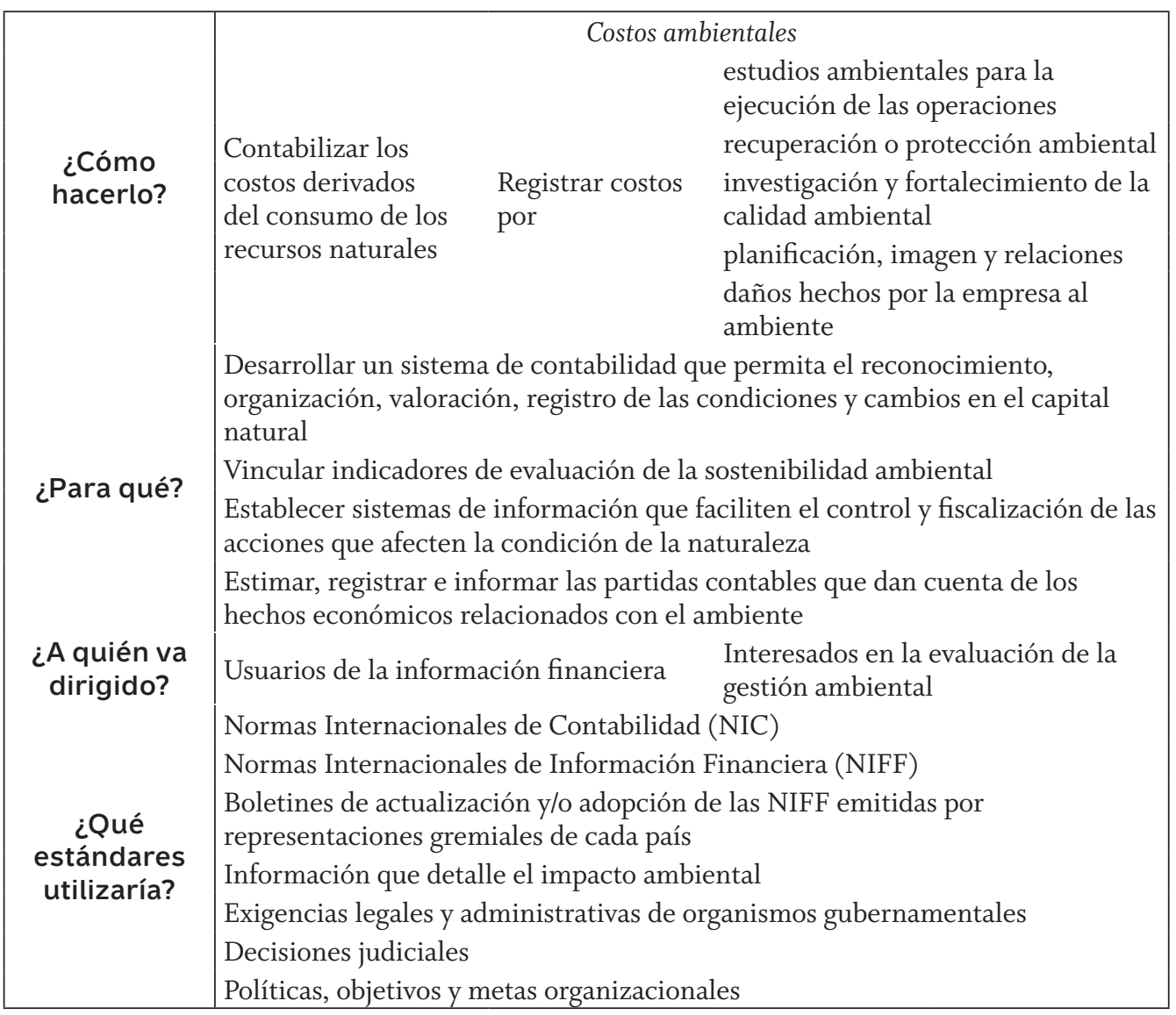

Cuadro 6. Sinopsis de la representación contable desde la perspectiva del impacto ambiental Fuente: elaborado propia (2014)

\section{A manera de reflexión final}

Con el propósito de suministrar información para la toma de decisiones y la presentación de los resultados económicos y financieros planteados originalmente por la contabilidad financiera, se deben incluir partidas que muestren los resultados económicos de las operaciones derivadas del mejoramiento, conservación y mantenimiento del ecosistema, en aras de contribuir al desarrollo sostenible de la población.
Esto representa un reto para la ciencia contable, la cual debe fundamentar con rigor sistemático el análisis, clasificación, registro, valoración y presentación de datos relevantes derivados de bienes e inversiones que tengan incidencia en el ecosistema, así como las obligaciones que proceden de efectos ambientales; de este modo, la tradicional estimación del patrimonio de las entidades deberá incluir en sus estados financieros partidas resultantes de la gestión ambiental o la valoración de la 
consecuencia de la explotación de la actividad económica cuyo efecto haya sido adverso a la conservación del ecosistema.

Desde esta perspectiva, diversos tratados y convenios internacionales han buscado la inclusión de partidas ecológicas a los reportes contables de las empresas, organizaciones e instituciones, entre sus objetivos se pueden destacar que han tratado de promover la conciencia por la preservación del medio ambiente por la actividad empresarial, la medición del impacto ambiental y el control de costos derivados de la preservación del ecosistema. Para ello, se fomenta la medición de variables ecológicas y la incorporación de evaluaciones ambientales dentro de la gama de reportes contables necesarios dentro de las entidades; su propósito se sustenta en conducir el efecto ambiental al lenguaje común de los negocios, al presentar entre sus rubros de cuentas valoraciones medio ambientales cuyos resultados podrían afectar, por un lado, la conservación de la flora y la fauna y, por otro, el supuesto de negocio en marcha establecido por la contabilidad en el cumplimiento de su objetivo.

A partir de la concepción ecológica, la representación contable contribuye mediante su lenguaje a la medición del efecto causado por la actividad industrial, a fin de promover la valoración de la contaminación ambiental, incentivar la fabricación de productos ecológicos, la utilización de maquinarias y equipos cuya incidencia en el hábitat sea reducida, la inversión en actividades de mejoramiento y conservación del ecosistema para finalmente alcanzar una conciencia empresarial ecológica fortalecida por el desarrollo ecológico de la población.
Desde la perspectiva de la contabilidad, la revelación de partidas referentes al ecosistema se ampara en el supuesto de negocio en marcha establecido históricamente por los principios contables y sustentado en los estándares internacionales de la contabilidad y la información financiera, los cuales hacen referencia a la hipótesis que los estados financieros que se presentan han sido preparados considerando que la entidad sobre la que se informa proyecta continuar su giro económico a largo plazo. Esta situación obliga a informar asuntos relevantes que puedan poner en duda este supuesto, los cuales desde la óptica ecológica podrían estar representados por obligaciones derivadas de la explotación económica con aprovechamiento de los recursos del ecosistema, sujetos a tipificaciones legislativas que pueden influenciar y determinar el desempeño empresarial de la entidad y exponerla a sanciones o al cese de sus actividades.

No obstante, la actividad industrial debe profundizar en el estudio, análisis y presentación de cifras que muestren el impacto negativo ocasionado por el desarrollo de su actividad productiva cuya incidencia en el ecosistema puede ser medido y revelado en los datos financieros de la entidad, con el objeto de satisfacer las demandas de información a los distintos usuarios de los reportes económicos y financieros de la compañía.

En este sentido, entre las partidas que pueden contemplarse para la representación contable del impacto ambiental, están los activos ambientales, los cuales permiten clasificar todo lo que constituya bienes, inversiones o propiedades, que sirven para la preservación, 
protección y recuperación ambiental. Por otra parte, en la gama del catálogo de cuenta de las organizaciones empresariales, los pasivos ambientales permitirán registrar aquellas deudas u obligaciones de la compañía, como consecuencia del impacto sobre el entorno físico, estas pueden ser derivadas de una naturaleza jurídica o tácita.

Asimismo, se estima conveniente registrar las provisiones cuando la empresa esté obligada, por normativa, a prevenir, reducir o reparar daños al ambiente, o cuando su política, objetivo, práctica del sector y las expectativas públicas lo consideren. Finalmente, puede contabilizar y llevar un registro sobre los costos ambientales, derivados del consumo de los recursos naturales, de la prevención, reducción y restauración de los impactos ambientales negativos de la actividad productiva realizada por una empresa.

Por este motivo, es necesario para las empresas desarrollar un sistema de información contable que, de manera cuantitativa y cualitativa, muestre datos relevantes para la evaluación, conocimiento y análisis de hechos referentes al ambiente. De este modo, las decisiones en materia de inversión, evaluación de operaciones y resultados contables trascenderán las fronteras del impacto económico y podrán enfatizar en el desarrollo sostenible de la actividad económica sobre la que se informa, cuyos fundamentos podrían incluir información de posibles daños ambientales, o promover el mantenimiento y mejora del ecosistema, siguiendo paradigmas integradores y de responsabilidad social del ente económico y el contexto de su organización productiva.

\section{Referencias}

Ablan-Bortone, Nayibe \& Méndez-Vergara, Elías (2004). Contabilidad y ambiente. Una disciplina y un campo para el conocimiento y la acción. Revista Actualidad Contable, Facultad de Ciencias Económicas y Sociales (FACES), Universidad de Los Andes (ULA), 7 (8), 7-22. Disponible en: http://www.redalyc. org/articulo.oa?id=25700802

Ariza-Buenaventura, Danilo (2000). Una perspectiva para captar la inserción contable en la problemática medio ambiental. Revista Legis de Contabilidad \& Auditoría, 4, 161-191. Barraza-Caro, Frank Eduardo \& Gómez-Santrich, Marta Eliana (2005). Aproximaciones a un concepto de contabilidad ambiental. Bogotá: Editorial Universidad Cooperativa de Colombia, EDUCC.

Blanco-Richart, Enrique (2006). Influencia de la nueva legislación en la información medioambiental suministrada por las empresas. Un estudio regional. Tesis doctoral de la Universidad Rey Juan Carlos, Madrid. Disponible en: http://dialnet.unirioja.es/servlet/ tesis?codigo $=2285$

Cañibano-Calvo, Leandro (2000). El medio ambiente en la contabilidad de las empresas. Revista Legis de Contabilidad \& Auditoría, 2, 81-96.

Comisión Mundial sobre el Medio Ambiente y el Desarrollo, WCED (1987). Nuestro futuro común. Documento en línea. Disponible en: http://www.upv.es/contenidos/ CAMUNISO/info/U0506189.pdf

Consejo de Normas Internacionales de Contabilidad (IASB) (2012). Norma 
Internacional de Contabilidad. Provisiones, Pasivos Contingentes y Activos

Contingentes (NIC-37). Disponible en: https://www.mef.gob.pe/contenidos/ conta_publ/con_nor_co/vigentes/nic/ NIC_037_2014.pdf

Fronti de García, Luisa \& Fernández-Cuesta, Carmen (2007). El Protocolo de Kyoto y los costos ambientales. Revista del Instituto International de Costos, 1, 9-31. Disponible en: http://www.revistaiic.org/articulos/num1/ articulo1_esp.pdf

García-Colín, Juan (2001). Contabilidad de costos. $2^{\text {a }}$ ed. México: Editorial McGraw-Hill.

Gómez-Villegas, Mauricio (2009). Tensiones, posibilidades y riesgos de la contabilidad medioambiental empresarial. Contaduría Universidad de Antioquia, 54, 55-78.

Disponible en: https://aprendeenlinea. udea.edu.co/revistas/index.php/cont/article/ viewFile/7963/7461

González-Martínez, Ana Citlalic (2001). Costos y beneficios ambientales del reciclaje en México. Una aproximación monetaria. $\mathrm{Ga}$ ceta Ecológica, 58, 17-26. México - México. Disponible en: http://www2.inecc.gob.mx/ publicaciones/gacetas/335/reciclaje.html Gray, Rob; Bebbington, Jan \& Walters, Diana (1999). Contabilidad y auditoría ambiental. Bogotá: Ecoe Ediciones.

Gray, Rob; Bebbington, Jan \& Walters, Diana (2006). Contabilidad y auditoría ambiental. Samuel Alberto Mantilla-Blanco (trad.). Bogotá: ECOE Ediciones.

Hansen, Don R. \& Mowen, Maryanne M. (2003). Administración de costos. Contabi- lidad y control. $3^{\mathrm{a}}$ ed., México: Ediciones Thomson, S.A.

Hernández-Sampieri, Roberto; Fernández-Collado, Carlos \& Baptista-Lucio, Pilar (1991). Metodología de la investigación. México: Editorial McGraw-Hill.

Horngren, Charles T.; Datar, Srikant M. \& Foster, George (2007). Contabilidad de costos: un enfoque gerencial. $12^{\mathrm{a}}$ ed., México: Pearson Educación S.A. de C.V., Prentice Hall.

Llull-Gilet, Antoni (2001). Contabilidad medioambiental y desarrollo sostenible en el sector turístico. Tesis doctoral, Universitat de les Illes Balears, UIB, Palma de Mallorca. Disponible en: http://www.tesisenxarxa.net/ bitstream/handle/10803/9419/talg1de1. pdf?sequence $=1$

Mantilla-Pinilla, Eduardo (2006). Desarrollo alternativo y contabilidad: una aproximación. Revista Legis de Contabilidad \& Auditoría, 25, 133-160.

Organización de Naciones Unidas, Asamblea General (1992). Cumbre para la Tierra +5, Río de Janeiro, 3-14 de junio de 1992. Documento en línea. Disponible en: http://www.un.org/spanish/conferences/ cumbre\&5.htm

Organización de Naciones Unidas, Asamblea General (2002). Cumbre Mundial sobre el Desarrollo Sostenible, Johannesburgo, Sudáfrica, 26 de agosto-4 de septiembre de 2002. Documento en línea. Disponible en: http://www.un.org/spanish/conferences/ wssd/, http://www.un.org/events/wssd/ summaries/envdevj35.htm

Organización de Naciones Unidas (2012). Contabilidad Ambiental y Económica Integrada. 
Manual de Operaciones. Estudios de Métodos. Manual de Contabilidad Nacional. Serie F No. 78. Disponible en: http://unstats.un.org/ unsd/publication/SeriesF/SeriesF_78S.pdf

Pacioli, Francesco di Luca, fray (1494). Summa de Arithmetica, Geometria, Proportioni et Proportionalità. Disponible en: http:// www.cervantesvirtual.com/obra/summade-arithmetica-geometria-proportioni-etproportionalita--2/

Quintero, Luis Eliel \& Anido, José Daniel (2004). Estimación de pasivos ambientales mediante la técnica Obligaciones Económicas de los Usuarios: Caso: Aguas de Mérida C.A. Revista Actualidad Contable Facultad de Ciencias Económicas (FACES). Universidad de Los Andes (ULA), 7 (9), 6177. Disponible en: http://www.saber.ula.ve/ bitstream/123456789/17339/1/articulo6.pdf Rueda-Delgado, Gabriel (2002). La contabilidad ambiental en el desarrollo sostenible. Revista Legis de Contabilidad \& Auditoría, 9, 11-128.

Rueda-Manzanares, Antonio; Aragón-Correa, Juan Alberto \& Martín-Tapia, Inmaculada (2006). La medición de las capacidades organizacionales de la empresa: validación de un instrumento de medida de la gestión medioambiental proactiva. Revista Española de Financiación y Contabilidad, REFC, 35 (130), 563-582. Disponible en: http://www. aeca.es/pub/refc/articulos.php?id=0234

Sabaté-Prats, Pere (2001). Problemática contable de los daños en el medioambiente. Caso del sector agrario. Revista Española de Financiación y Contabilidad, REFC, 30 (110), 9731000. Disponible en: http://aeca.es/pub/ refc/acceso.php?id=0135
Scavone, Graciela (2002). Contabilidad de gestión ambiental. La producción limpia como oportunidad de negocio. Ponencia presentada en la Facultad de Ciencias Económicas, Universidad de Buenos Aires, UBA. Disponible en: http://www2. medioambiente.gov.ar/documentos/ ordenamiento/tercer_seminario_ internacional/scavone.pdf

Tejada-Ponce, Ángel (1999). La gestión y el control socio-económico de las interacciones empresa-medio ambiente. Contribuciones de la contabilidad a la gestión sostenible de la empresa. Madrid: Instituto de Contabilidad y Auditoría de Cuentas, ICAC.

Tua-Pereda, Jorge (2001). Normas Internacionales de Contabilidad y Auditoría sobre la incidencia del medio ambiente en la información financiera. Revista Legis de Contabilidad \& Auditoría, 7.

- Fecha de recepción: 12 de marzo de 2015

- Fecha de aceptación: 11 de mayo de 2015

- Disponible en línea: 30 de agosto de 2015

\section{Para citar este artículo}

Colmenares, Loyda; Adriani, Rolando \& Valderrama, Yosman (2015). Representación contable desde la perspectiva del impacto ambiental empresarial. En el contexto del desarrollo de actividades industriales en Latinoamérica. Cuadernos de Contabilidad, 16 (41), 259-280. http://dx.doi. org/10.11144/Javeriana.cc16-41.rcpi 